\title{
Intermédialités
}

Histoire et théorie des arts, des lettres et des techniques

Intermediality

History and Theory of the Arts, Literature and Technologies

\section{Impressions et figurations du visage dans quelques films de Chris Marker}

\section{André Habib}

Numéro 8, automne 2006

Envisager

Facing

URI : https://id.erudit.org/iderudit/1005545ar

DOI : https://doi.org/10.7202/1005545ar

Aller au sommaire du numéro

Éditeur(s)

Centre de recherche sur l'intermédialité

ISSN

1705-8546 (imprimé)

1920-3136 (numérique)

Découvrir la revue

Citer cet article

Habib, A. (2006). Impressions et figurations du visage dans quelques films de Chris Marker. Intermédialités / Intermediality, (8), 153-171.

https://doi.org/10.7202/1005545ar
Résumé de l'article

Le visage dans les œuvres de Chris Marker se présente comme l'objet d'une recherche sur l'image et sur la prégnance de la mémoire. Il est également le lieu d'un déchiffrement, s'offrant au regard tout en se dérobant sous des masques, entre les rets du temps. L'hypothèse de cet article est que le visage nous offre un fil de lecture pour aborder l'oeuvre de Marker : il y apparait comme une figure de médiation à part entière, qui en livre les promesses et les apories, les dissimulations et les instants de vérité. 


\title{
Impressions et figurations du visage dans quelques films de Chris Marker
}

\author{
André HablB
}

L

es impressions les plus prégnantes, les plus durables, sont parfois aussi les déraisonnable sur la pensée, marquée et approfondie par l'expérience. Elles ne font pas toujours appel à un appareillage conceptuel décidé d'avance, doté de problématiques lisses, de développements bien échelonnés. Elles ne possèdent d'évidence, en premier lieu, que pour celui qui a décidé qu'il y avait là quelque chose. Ce type d'impressions fait travailler l'imagination à partir de ce qui s'est imprimé dans la mémoire'.

Il en va de la sorte pour ce qu'il faut bien appeler la «figure du visage» dans les films de Marker: il y a, en effet, quelque chose là, et qui insiste, et qui, pour qui s'y attarde, confine rapidement au vertige. Le nombre d'occurrences et d'exemples significatifs dans les films rend problématique l'imposition de balises: où commence et où finit le critère de pertinence pour aborder cette figure? N'est-elle pas potentiellement infinie, dans la mesure où on retrouve chez Marker beaucoup de visages? Au-delà de ce constat, que peut-on dire de la figure - ou des figures - du visage chez Marker? Possède-t-elle des traits singuliers? En somme, comment peut-on la traiter, en partant de ces impressions que ses films ont déposées en nous²?

1. Cette terminologie fait volontairement écho à la riche théorisation de la «figure » chez Martin Lefebvre, Psycho: de la figure au musée imaginaire, Paris, Montréal, L'Harmattan, 1997.

2. Cette figure du visage chez Marker a été abordée dans un très bel article dont je ne connaissais pas l'existence au moment d'écrire ce texte. L'auteur analyse les visages chez Marker principalement à partir des écrits de Benjamin sur la photographie. Largument m'a paru suffisamment distinct de celui que je propose pour ne pas y consentir une place plus importante. Voir Catherine Gillet, «Visages de Marker », Théorème, Philippe Dubois (dir.), «Recherches sur Chris Marker», Paris, n 6, 2002, p. 74-82. 
Le visage dans les films de Marker apparaît comme le lieu et l'objet d'une recherche sur l'image et la mémoire. Le visage est aussi l'occasion d'un déchiffrement. Il s'offre à la lecture, tout en se dérobant sous des masques, entre les rets du temps. Il joue le rôle d'intercesseur fragile entre les vivants et les morts, pousse le dispositif technique à se révéler, lorsque le regard croise celui de la caméra. Perte, deuil et fragilité, défi au regard et lutte entre les images, prophétie et avertissement, le visage, chez Marker, dit tout cela. Ce sont tous ces visages de femme, de Sans soleil (1982) à Level Five (1996), ces visages torturés des prisonniers, des cadavres, des écorchés de la guerre (La jetée, 1962, Level Five, Souvenir d'un avenir, 2002); ce sont aussi ces séries de masques africains (Les statues meurent aussi, 1953), japonais (Sans soleil) ou virtuels (Level Five), qui invoquent, multiplient ou délient les facettes du visage. À l'évidence, le visage apparaît chez Marker de façon suffisamment riche et insistante pour réclamer une «autonomie» figurative. L'hypothèse serait qu'il nous offre, pour cette raison, un fil de lecture pour traverser cette œuvre plurielle.

La figure du visage dans ses films possède sa propre logique, que cet article s'emploiera transversalement à retracer en suivant certaines questions qu'elle soulève. Ce qu'il s'agira de démontrer c'est que le visage est une figure de médiation privilégiée dans l'œuvre de Marker, qui en livre les promesses et les apories, les dissimulations et les instants de vérité.

\section{LE VISAGE, POUR L'HISTOIRE}

Comme l'a excellemment montré Jacques Aumont ${ }^{3}$, le visage offre un axe privilégié pour traverser l'histoire du cinéma: du cinéma des origines à la mise en forme des codes classiques, de ses ruptures modernes jusqu'à sa déréliction, sous le poids de la publicité et de la mass-médiatisation actuelle. La «pratique» du visage chez les grands auteurs du cinéma constituent des étapes décisives qui permettent de révéler des aspects fondamentaux de la relation de cet art au monde. Il est par ailleurs significatif qu'il y ait eu une telle abondance de réflexions théoriques sur le visage au cinéma, comme s’il se jouait là quelque chose de décisif pour cet art en entier : en effet, le visage révèle, selon Aumont, « une idéologie des pratiques figuratives qui se caractérise par trois préoccupations majeures: le réel, l'expression, le temps ${ }^{4} »$. Autant dire, tout le cinéma.

3. Jacques Aumont, Du visage au cinéma, Paris, Éditions de l'Étoile, Cahiers du cinéma, 1992.

4. Jacques Aumont, Du visage au cinéma, p. 168. 
Aborder le visage, c'est aussi envisager sa place singulière dans les films5. Nous pourrions dire, à la suite de Deleuze, que le visage y fonctionne sur deux modes essentiels ${ }^{6}$ : soit sur le mode du raccord, soit, valant pour lui-même, comme entité autonome. Le visage apparaît soit comme le lieu par excellence de l'identification, de la focalisation et de l'articulation des unités narratives (raccord dans l'axe du regard, intensification, marques de ponctuation, etc.) ; soit il apparaît comme ce qui s'arrache momentanément au récit pour exprimer un «pur affect non actualisé». C'est peut-être ce qu'il faut appeler, à la suite d'Epstein, la «photogénie», le glamour éclatant de la star ou la stase du «portrait», dans lequel le visage de l'acteur apparaît soudain au travers du masque du personnage: dans chacun de ces cas, le film cesse de fonctionner sur un mode narratif, et se met à opérer sur un mode d'intensité spectaculaire ${ }^{7}$. Nous dirons alors que le visage est soit une puissance d'arrêt de la narration - ce serait son « mode spectaculaire» - , soit une instance de raccord du récit - ce serait son « mode narratif ${ }^{8} »$. Ces deux types peuvent tout à fait coexister, et ne cessent d'ailleurs de se relayer dans un même film.

\section{MODALITÉS DU VISAGE CHEZ MARKER : VISAGE-SOUVENIR}

Qu'en est-il chez Marker? Son cinéma semble échapper, au premier abord, à ce genre de typologie. En y regardant de plus près, toutefois, on pourrait retracer ces

5. Nous sommes forcé de contourner la question du "gros plan », qui possède une très longue tradition théorique, et sur laquelle notamment Deleuze s'est exprimé dans une célèbre formule : «le gros plan, c'est le visage [...] le visage, c'est le gros plan. » (Gilles Deleuze, Cinéma 1. L'image-mouvement, Paris, Éditions de Minuit, coll. «Critique», 1983, p. 125) Sur cette question toutefois, à titre indicatif, on se référera à Pascale Bonitzer, «La métamorphose », dans Décadrages, Paris, Cahiers du cinéma, Éditions de l'Étoile, 1995 [1984], p. 87-91, ainsi qu’à Béla Balàzs, L'esprit du cinéma, trad. J. M. Chavy, Paris, Payot, 1977.

6. Deleuze propose de distinguer un «visage intensif» et un «visage réflexif ou réfléchissant»: «nous nous trouvons devant un visage intensif chaque fois que les traits s'échappent du contour, se mettent à travailler pour leur compte et forme une série autonome qui tend vers une limite ou franchit un seuil [...] Au contraire, nous sommes devant un visage réflexif ou réfléchissant tant que les traits restent groupés sous la domination d'une pensée fixe ou terrible, mais immuable et sans devenir, éternelle en quelque sorte. » (Gilles Deleuze, Limage-mouvement, p. 128-129)

7. Jacques Aumont, Du visage au cinéma, p. 58-64; p. 127-136.

8. Cette distinction entre un «mode narratif» et un «mode spectaculaire» du visage s'apparente sur bien des points à la réflexion que propose Martin Lefebvre sur le paysage au cinéma. Voir Martin Lefebvre, «Entre lieu et paysage au cinéma », Poétique, $\mathrm{n}^{\mathrm{o}} 130$, avril 2002, p. 138 . 
deux modalités du visage, mais traduites dans les termes de son auteur, en rompant avec le cadre régulier du cinéma narratif classique: si le visage se manifeste chez Marker sur le mode «spectaculaire», c'est comme puissance d'arrêt d'un temps, soustrait à l'enchaînement, et surtout marqué par la cicatrice d'un souvenir. Le visage peut également se présenter sur le mode du « raccord», comme instance d'embrayage d'une série, permettant d'articuler ou de réenchaîner une «fiction de mémoire ${ }^{9}$ ".

La jetée, l'éblouissant photomontage que Marker réalise en 1962, se déploie tout entier à partir du visage d'une femme, vu par le héros un dimanche, à Orly, peu de temps avant le déclenchement d'une troisième guerre mondiale qui allait rendre la vie sur terre invivable. L'homme «dont on raconte l'histoire », aperçoit ce visage de femme quelques instants avant d'être le témoin d'un meurtre.

156 Ce meurtre se révélera, dans la boucle de la fiction, être l'image de sa propre mort. Le personnage, nous apprend le narrateur, se demandera longtemps si cette image était bel et bien un souvenir, ou si elle n'était pas plutôt le produit de son imagination, désireuse d'une image du bonheur en temps de paix, après le spectacle des horreurs qu'il avait traversées. Si le bonheur s'incarne dans un visage de femme, l'horreur de la guerre est sans visage, elle ne présente que des ruines. Mais ces deux images juxtaposées, ces deux «faces » de la réalité, sont le lieu d'une tension féconde, que l'on retrouvera dans toute son œuvre: le visage d'une jeune femme peut côtoyer le visage de soldats émaciés (comme dans son installation vidéo Prelude: The Hollow Men, 2005), la photographie de la star, celle d'une lépreuse (comme dans Souvenir d'un avenir).

Dans La jetée, c'est le souvenir de cette femme, empreinte mouvante et insaisissable, et l'obsession qu'elle suscite qui rend le prisonnier-cobaye apte à voyager dans le temps. Si les scientifiques qui l'ont capturé veulent l'y envoyer, c'est afin qu'il ramène du futur une source d'énergie qui permettra aux hommes de survivre; lui, il y plonge pour rencontrer dans le passé cette femme, qu'il retrouvera, par bouffées brèves, aux Tuileries, au Musée de l'Homme, etc. Leurs rencontres culmineront dans un instant d'intimité, qui leur sera aussitôt dérobé. La femme semble allongée sur un lit, son visage est cadré en gros plan. Se succèdent alors une série de photos, fondues l'une dans l'autre, de son visage, de plus en plus rapprochées, comme s'il allait s'animer, jusqu'à ce que, soudain, 24 photogrammes consécutifs, en une seconde, défilent sous nos yeux, nous donnant à voir l'image soudain «animée» d'un clignement de paupières, adressé directement à la caméra (figs. 1-2). Cet Augenblick échappe à la règle «photographique» du film, de telle sorte que l'on assiste à une interruption et une réactivation du

9. Jacques Rancière, «La fiction documentaire: Marker et la fiction de mémoire», dans La fable cinématographique, Paris, Éditions du Seuil, 2001, p. 201. 
IMPRESSIONS ET FIGURATIONS DU VISAGE DANS QUELQUES FILMS DE CHRIS MARKER
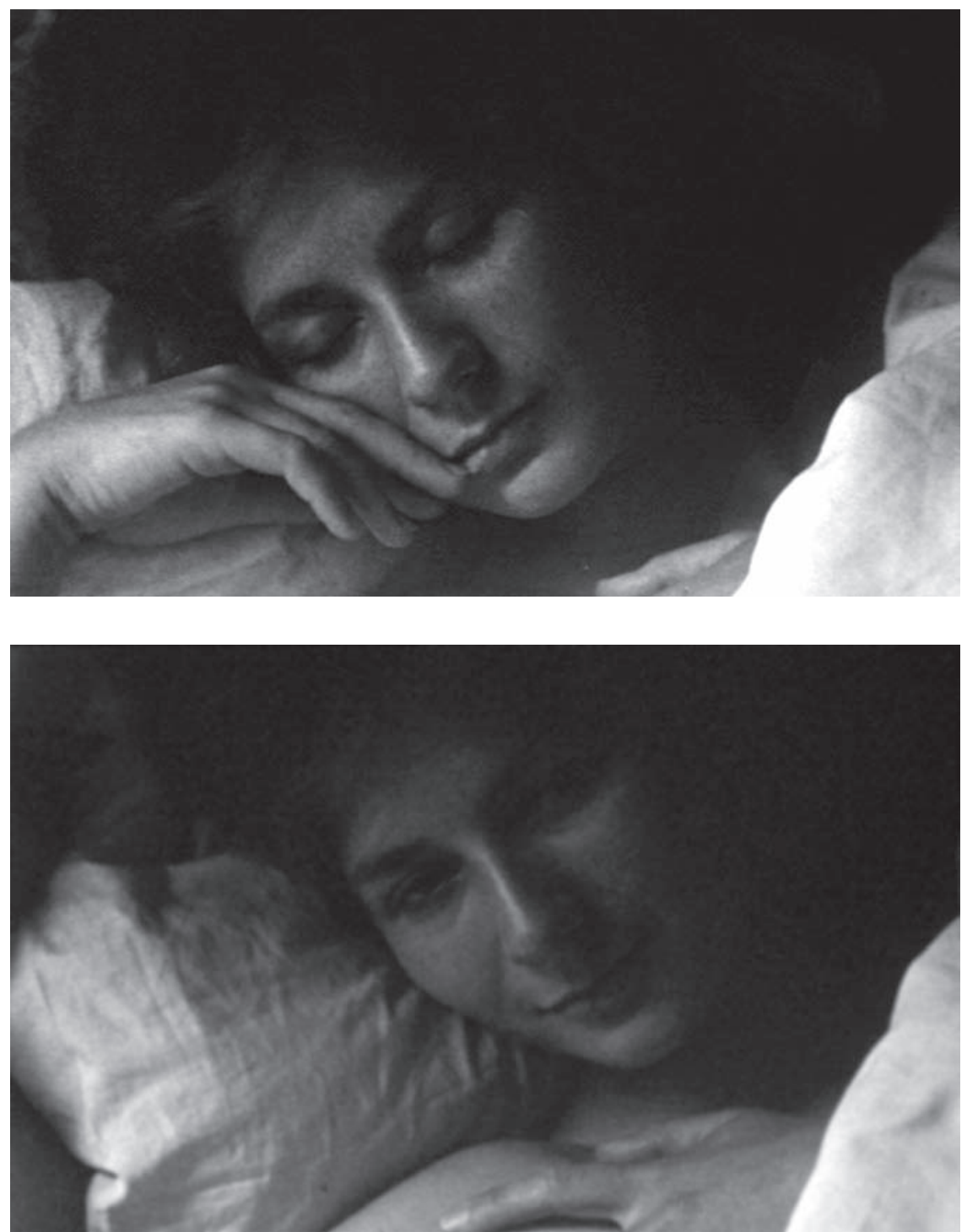

Figs. 1-2. Chris Marker, La jetée, 1963 (C) Argos Films. Avec l'aimable autorisation de Argos Films. 


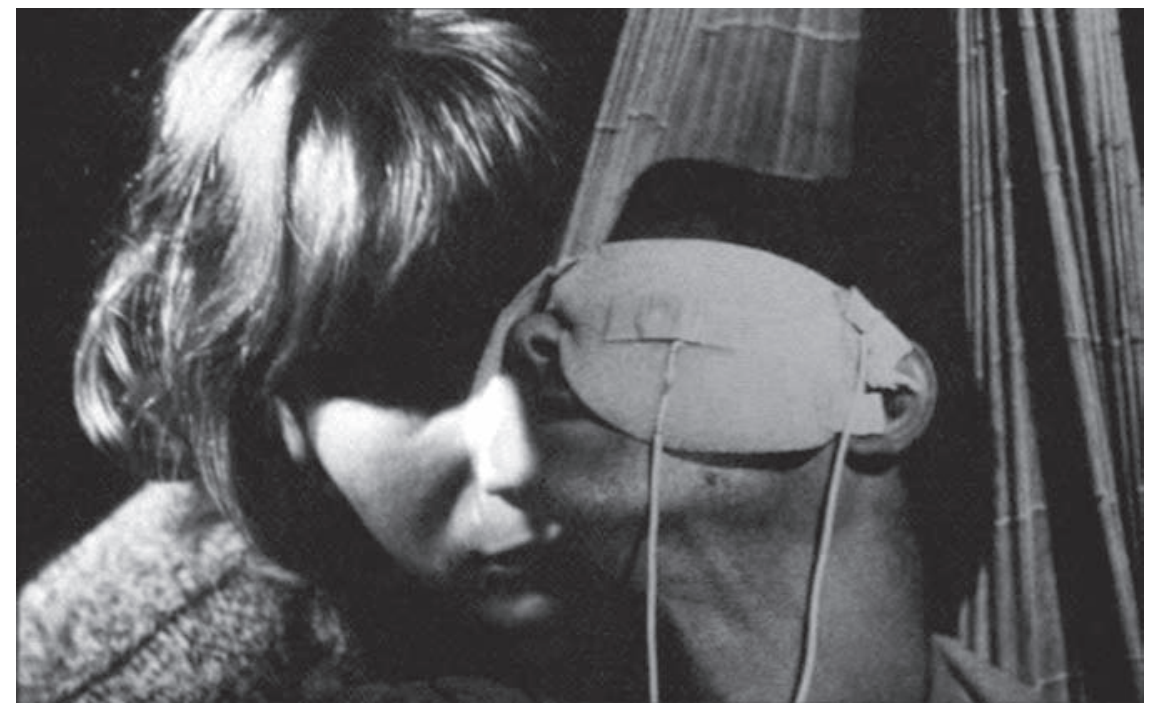

Fig. 3. Chris Marker, La jetée, 1963 (c) Argos Films. Avec l'aimable autorisation de Argos Films.

temps. Le visage de la femme entaille et reprise le temps, y imprimant un nouvel instant de bonheur.

Si l'obsession du héros pour «cette image du bonheur» le prédisposait au voyage dans le temps, c'est aussi elle qui le condamnera à mort. Lorsque les hommes du futur lui donnent la possibilité de retourner dans le temps, il choisit l'instant précis, sur la jetée d'Orly, où le visage s'était, pour la première fois, empreint dans sa mémoire. Courant à la rencontre de la femme, il sera abattu: «on n’échappe pas au temps ${ }^{10}$ ». Le visage de la femme est donc la condition d'articulation du récit (sa trame, le principe qui le boucle), et en même temps son arrêt, sa suspension (instant de la mort, empreinte dans la mémoire, arrachement au récit). Plus encore, ce visage de femme est agencé visuellement - dans une série de surimpressions somptueuses (fig. 3) - avec le visage torturé du prisonnier : ce dernier doit souffrir pour l'atteindre. Même lorsqu'il est «pris pour lui-même », le visage est pris dans une série de connexions, entre la voix du narrateur, la succession des plans, la toile du récit. Le travail sur le visage n’exprime pas un quelconque processus d'individuation ou d'expression d'intériorité. Il traduit plutôt une série d'affects intensifs : traits de souffrance, marque du souvenir, éclair de bonheur, aveuglement du désir.

10. Pour mémoire, on se souviendra que l'homme est tué sur la jetée d'Orly par les scientifiques du présent, qui sont allés le chercher dans le passé pour l'assassiner après s'être servis de lui. 


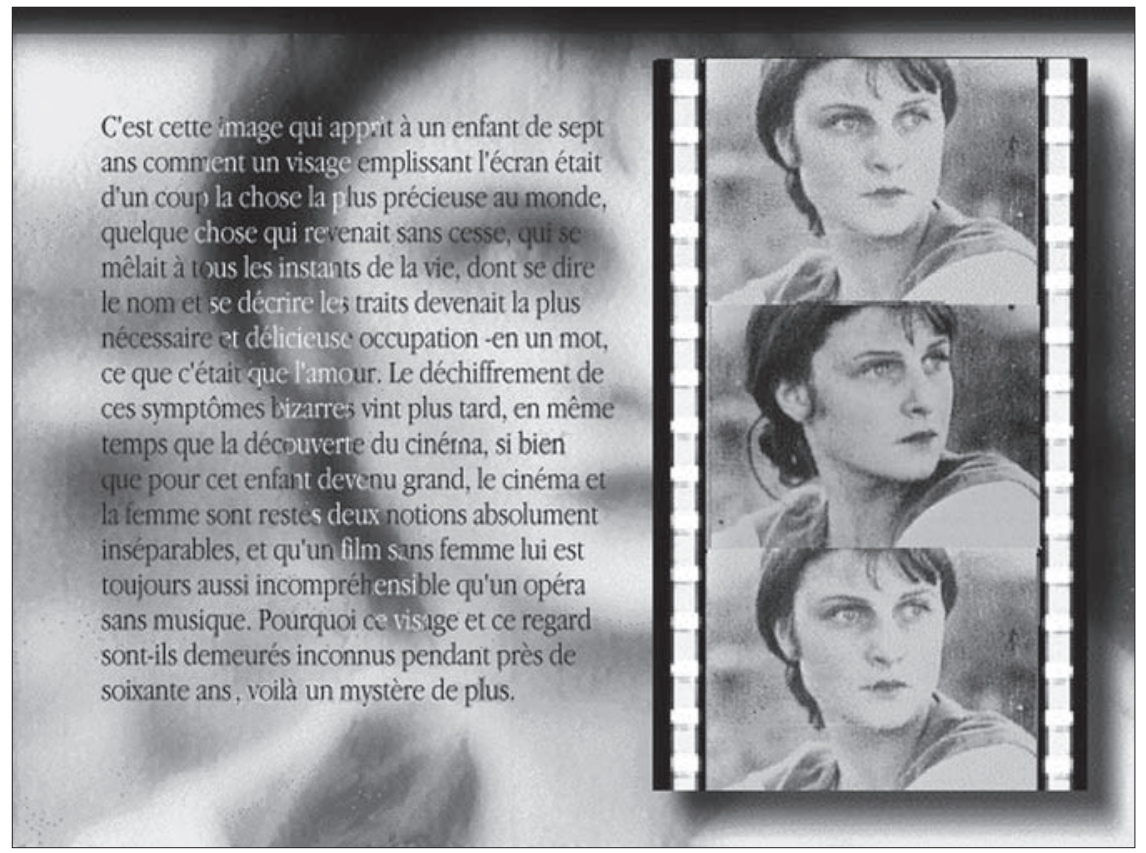

Fig. 4. "C'est cette image qui apprit... », page extraite du CD-Rom Immemory One, Centre Georges Pompidou, 1997. (C) Chris Marker / Centre Georges Pompidou.

\section{SIMONE GENEVOIS, PREMIÈRE IMPRESSION}

La figure du visage, chez Marker, est liée à une empreinte dans la mémoire. Si l'on a pu dire que ses films participent tous, à différents degrés, de l'autoportrait (chaque film serait un «album de famille», une correspondance, un tombeau pour l'ami, Tarkovsky, Medvedkine ${ }^{11}$ ), nous pourrions faire remonter cette prégnance du visage, dans son œuvre, à un certain «souvenir personnel» - bien qu'il soit impossible de savoir s'il est véritable ou fictif.

Dans la «Zone cinéma » de son CD-Rom Immemory One, Marker relate une série de «premières impressions » cinéphiliques. On y retrouve, parmi d'autres un «montage » texte-image dédié à l'actrice française Simone Genevois, aujourd'hui disparue des mémoires, qui avait joué dans un film de Marc de Gastyne, La merveilleuse vie de Jeanne d'Arc (1927). Sur la droite de l'écran, trois photogrammes apparaissent enfilés à la verticale, sur une bande de pellicule (fig. 4). À la gauche, on peut lire:

11. Voir sur ce point Viva Paci, «"Je vous écris d'un pays lointain”, Le tombeau d'Alexandre de Chris Marker », Sociétés et représentations, «La croisée des médias», nº 9 , avril 2000, p. 249-258. 
C'est cette image qui apprit à un enfant de sept ans comment un visage emplissant l'écran était d'un coup la chose la plus précieuse au monde, quelque chose qui revenait sans cesse, qui se mêlait à tous les instants de la vie, dont se dire le nom et se décrire les traits devenait la plus nécessaire et délicieuse occupation - en un mot, ce que c'était que l'amour.

Cette image est lourde de signification. Elle traduit le caractère éminemment privé de son expérience des images (une première image de cinéphile) ${ }^{12}$. Elle se présente sous le signe de l'obsession, elle contamine le temps présent, revenant «sans cesse », se mêlant «à tous les instants de la vie». Le visage, présent parce qu'absent, posséderait ainsi, tel qu'il nous le livre ici, un caractère premier, commandant en retour toute une pratique du regard et un travail sur la mémoire. Le visage est ce qui se dérobe, et, en même temps, ce à quoi on ne peut échapper et qu'il faut retenir, précisément parce qu'on est retenu par lui.

C'est sans doute dans cet écart entre la perte de l'objet familier, de l'objet d'amour, et l'urgence, voire le devoir d'y revenir, de le faire revenir, qu'on touche au plus près l'enjeu de la médiation dans les films de Marker ${ }^{13}$. On comprend dès lors la place qu'y représente la figure du visage, à la fois comme objet d'une mémoire et signe d'une mémoire: se rappeler un visage, c'est lui reconnaître une place comme objet dans sa mémoire, mais c'est aussi se rappeler que ce visage possède une mémoire (les tisseuses du marché d'Okinawa, le Iakoutsk de Sibérie, les femmes du marché de Bessao).

\section{L'AUTOPORTRAIT MARKERIEN}

Chaque film de Marker, nous le disions, serait une forme d'autoportrait. Mais à la différence de Boris Lehman (Babel: lettre à mes amis restés en Belgique, 1991), Jonas Mekas (Diaries, Notes and Sketches, 1969), Agnès Varda (L'Opéra-Mouffe, 1958, ou Les glaneurs et la glaneuse, 2000), voire Jean-Luc Godard (JLG/JLG: autoportrait en décembre, 1994), qui ont réalisé des autoportraits où ils se mettent en scène, Marker, c'est connu, ne paraît jamais à l'écran, on ne sait pas à quoi

12. Voir le très beau texte de Marker qui accompagnait son installation Silent Movie: Chris Marker, «The rest is silent», Trafic, n 46, été 2003, p. 57-62.

13. « [Marker] tranforms the faces of strangers around the world momentarily, fragmentarily, into so many masks-death masks and primal faces of women. These faces ever call to mind the same face: the face, familiar but now lost, of the mother or the beloved. A face that is familiar because lost, and thus always recovered in the multitude of images. It is then, in full media frenzy that mediation once again becomes possible.» (Johanne Villeneuve, «Utopian Legacies: Memory, Mediation, Cinema », dans Brian Neville et Johanne Villeneuve [dirs.], Waste-Site Stories: the Recycling of Memory, Albany, State University of New York Press, 2002, p. 208-209) 
ressemble son visage, au mieux saurait-on reconnaître sa voix pour l'avoir entendue dans quelques rares films (À bientôt j'espère, 1968, Le mystère Koumiko, 1965, Le fond de l'air est rouge, 1977, Level Five). N'est-il pas alors singulier de parler de la figure du visage et d'une pratique de l'autoportrait chez un auteur dont on ne possède, à tout prendre, aucune photo?

Une exception semble vouloir confirmer la règle. Une photographie de l'artiste apparaît en effet sur la quatrième de couverture de son livre Commentaires ${ }^{14}$. Cet autoportrait photographique montre un visage caché derrière un appareil photo à deux objectifs, tenu par deux mains. La photo semble avoir été prise dans un miroir. On voit clairement un œil droit nous/se fixant. Le reste de l'image est plongé dans l'obscurité. Cette photo nous fait comprendre dans quel sens il faut aborder l'auto de l'auto-portrait ${ }^{15}$. C'est la caméra et ce qu'elle photographie (c'està-dire elle-même et l'autre) qui tiennent lieu du visage autoportrait. L'autoportrait passe par une technique qui en médiatise à la fois le contenu et la forme réfléchie. Le visage de Marker a beau être voilé, la technique, le dispositif photographique (et éventuellement filmique), révèle, en se montrant, son «vrai »visage ${ }^{16}$.

Du coup, Marker nous rappelle que, si l'accès au visage suppose un certain rapport de médiation, c'est que, d'une certaine façon, le visage est toujours médiation. Sa représentation nécessite un détour qui tient compte de l'interface technique et symbolique du médium. Pour cette raison, toute image du visage est en décalage, elle ne saurait être parfaitement adéquate à ce qu'elle vise, son accès n'est jamais immédiat. Mais c'est peut-être dans ce passage, ce détour, cette dissimulation, que se trouve la vérité du visage, que ce dernier peut se dévoiler. Le visage de Chris Marker, après tout, ne devient-il pas «reconnaissable » grâce à tous ces visages, captés au fil de ses voyages? N'est-ce pas en passant par eux que son regard se révèle? N'est-ce pas eux qui nous offrent la clé de composition de son autoportrait?

14. Chris Marker, Commentaires, Paris, Éditions du Seuil, 1961. On pourrait bien entendu nous objecter que rien ne garantit qu'il s'agit de Marker sur cette photo, que ce n'est pas nécessairement son œil qui nous regarde, que ce n’est pas sa main qui tient l'appareil, son visage qui se dissimule, mais « un œil », « une » main, « un »visage. Si rien, en effet, ne nous permet de garantir l'identité de celui qui s'est auto-photographié, le dispositif de dissimulation et la mise en scène du processus de «médiation » dans lequel il apparaît, disent bien de quelle façon il nous faut envisager la représentation du visage du cinéaste.

15. Sur cette question, voir Muriel Tinel, «Le cinéma et l'autoportrait: de l'expression de soi à l'expérience d'un support », Hors champ, avril 2006, http ://www.horschamp. qc.ca/article.php3?id_article $=220$.

16. Une œuvre de Michael Snow, Authorization (1968), s'employait aussi à une telle mise en abyme de la photographie, de la caméra, du photographe et du miroir. 


\section{PARADOXES DU DÉCHIFFREMENT OU QUE PEUT UN VISAGE?}

Si le visage est un objet privilégié de la mémoire dans les films de Marker, chaque visage est aussi le siège et le signe d'une mémoire dont la lecture et l'appréhension sensible passent par la technique audiovisuelle. La technique nous permet en effet de traiter le visage comme objet de mémoire («je me rappelle ces visages-là ») ou signe d'une mémoire («derrière chaque visage se cache une mémoire»). Ce serait la promesse. Mais en même temps, que nous dit un visage? De quoi dépend sa lecture? À quelle intériorité nous donne-t-il accès? Ce serait sa question. La figure du visage, chez Marker, naît de la confrontation entre cette promesse et cette question.

Déchiffrer un visage, cela peut vouloir dire en libérer les traits, le faire signifier au-delà de son temps historique. C'est à cela que s'emploie Le souvenir d'un avenir (2002), film coréalisé avec Yannick Bellon, à partir des photographies - et sur la vie - , de Denise Bellon, une photographe ayant côtoyé, notamment, le groupe des surréalistes dans les années 1930. Ses photographies, tout particulièrement celles de l'entre-deux-guerres, nous permettent de déchiffrer le passé en y lisant les traces de l'avenir, logées dans leur présent-passé. Ce que l'on lit sur ces visages, celui de Marcel Duchamp, de Pablo Picasso, des étudiants de la Sorbonne, des visages défigurés de la première guerre, c'est, anachroniquement, tout ce que ces visages, inquiets, souriants, terrifiés, ne savaient pas encore qu'ils allaient voir. Il s'agit de montrer un passé qui déchiffre un avenir, de montrer comment un après-guerre, pour eux, devient un avant-guerre, pour nous qui, aujourd'hui, les regardons. Denise Bellon témoigne par ses photographies d'un avenir dont le temps nous a permis de lire les traits en les déployant sur le grand écran de l'histoire. C'est le cas de ces soldats défigurés, «rescapés de la grande guerre», qui «se réunissent, $[. .$.$] se montrent, [. .$.$] montrent à tous ce que la$ guerre peut faire aux hommes, pour témoigner, pour avertir. » Et le commentaire ajoutera: «on sait la suite». En même temps, ces «gueules cassées» peuvent évoquer pour nous ces visages que peindra plusieurs années plus tard Francis Bacon; elles nous rappellent les tableaux cubistes qui inauguraient un état défait du visage dans l'art moderne, et qui avaient rencontré, auparavant, le «primitivisme » des masques africains, inséparable d'un certain «visage » du colonialisme (que Denise Bellon a également photographié, en Algérie notamment), etc. Nous le disions, les visages nous lisent l'histoire, en nous permettant de lier ces moments disparates...

\section{SÉRIES-VISAGES}

Les grands documentaristes - Vertov, Wiseman, van der Keuken, Ivens, Perrault, Groulx - ont tous compris qu'un visage est un signe particulièrement riche, 
parce que, précisément, il est le siège de signes innombrables. Chaque visage, pourvu qu'on s'y applique, documente un regard du temps, c'est-à-dire un raccord entre soi et le monde. Dans Sans soleil, la narratrice nous dit: « [S] ous chacun de ces visages, une mémoire. Et là où on voudrait nous faire croire que s'est forgée une mémoire collective, mille mémoires d'hommes qui promènent leur déchirure personnelle dans la grande déchirure de l'histoire. » Il est certes impossible de rendre ces «mille mémoires » d'hommes; il serait toutefois possible d'en présenter «la déchirure personnelle », la suture paradoxale, qui raccorde ce visage et ce regard à l'histoire, à d'autres mémoires, à d'autres images, à une multiplicité.

À la fin du Joli mai, Marker s'adressait aux visages d'une foule parisienne, dont la caméra de Pierre Lhomme captait toute l'inquiétude: «Que se cache sous vos visages [...] Avez-vous peur des fantômes? » Cette question, il se la posera plusieurs années après, aux visages assoupis des passagers du métro de Tokyo de Sans soleil, ou aux vieilles tisseuses du marché d'Okinawa de Level Five. Les trois exemples que nous venons de citer sont fort révélateurs et méritent que nous nous y attardions.

Dans Le joli mai, ces visages des passants, portant, nous dit le commentaire, leur "prison à l'intérieur d'eux", se connectent au sans visage des prisonniers dans leur cellule, dont on a entendu précédemment la voix, et à la grisaille du paysage parisien, dans une triple capture, qu'agence, comme sur une partition, la voix du narrateur. La question n'est plus «à quoi pensent-ils », mais à quelles pensées ces visages permettent la circulation? La voix et le montage décomposent littéralement les traits des ces visages en leur imprimant une peur sourde et muette, qui les désindividualise, les dissémine en une collectivité de spectres inquiets: c'est le visage de Paris, en 1962, lu par Marker.

Dans Sans soleil, les lignes de métro de Tokyo deviennent des fils invisibles qui raccordent les rêves des voyageurs dans un grand film immense et impossible, défilant à toute vitesse. Marker nous montre les visages des travailleurs, des femmes, des vieillards, des enfants endormis, emportés par le tracé lumineux des trains, entre lesquels il insère des images de films japonais, visages de fantômes, visages de samouraïs, visages de personnages manga. Ce n'est pas seulement alors la projection imaginée des rêves des voyageurs. C'est un agencement qui se produit entre les visages des voyageurs et les visages d'un rêve collectif, lui-même déterritorialisé à l'intérieur du réseau du métro, lui-même inscrit dans un film-rêve, etc.

Dans Level Five, une très belle séquence s'ouvre et se clôt sur les visages des femmes du marché d'Okinawa, qui sont, nous dit le commentaire, les «gardiennes de sa mémoire». Pour lire ces visages, pour leur donner toute leur intensité, Marker les agence avec une série d'évocations et d'images des corps torturés de la bataille, du musée qui essaie de raconter, sans jamais y parvenir, l'horreur d'Okinawa, des photographies des jeunes infirmières de Shoari mortes suicidées 
dans leur grotte; des visages des touristes armés de leurs appareils-photos, etc. La série raccorde les visages sur autre chose que lui, que le mystère de ces visages de femmes du marché, porteurs d’une mémoire invisible, a permis de déclencher.

Tout cela existe sur un même plan de consistance qui rappelle ce mot de Deleuze et Guattari, dans Mille plateaux:

[...] chaque trait libéré de visagéité fait rhizome avec un trait libéré de paysagéité, de picturalité, de musicalité, non pas une collection d'objets partiels, mais un bloc vivant, une connexion de tiges où les traits d'un visage entrent dans une multiplicité réelle, dans un diagramme ${ }^{17}$.

\section{REGARDS-CAMÉRA}

Nous disions qu'il y a, chez Marker, deux grands modes de représentation du visage: soit comme instance de raccord à l'intérieur d'une série, soit comme puissance d'arrêt du temps.

Dans Sans soleil, cette puissance d'arrêt du temps est mise en évidence dans le jeu de regard avec la caméra, qui suspend le temps précisément parce qu'il force la caméra et son regard à se montrer. Il est clair qu'il ne s'agit pas seulement de montrer le regard de l'autre, mais de «soutenir le regard». Soutenir le regard, c'est accepter d'être exposé par lui. C'est d'ailleurs une des questions centrales de Sans soleil:

Comment filmer les dames de Bissau? Apparemment, la fonction magique de l'œil jouait là contre moi. C'est sur les marchés de Bissau et du Cap-Vert que j’ai retrouvé l'égalité du regard, et cette suite de figures si proches du rituel de la séduction : je la vois - elle m'a vu - elle sait que je la vois - elle m'offre son regard, mais juste à l'angle où il est encore possible de faire comme s'il ne s'adressait pas à moi - et pour finir le vrai regard, tout droit, qui a duré $1 / 25^{\mathrm{e}}$ de seconde, le temps d'une image ${ }^{18}$.

Le «vrai regard", à ce moment, est celui qui s'adresse directement à la caméra, et qui ne dure, effectivement, à l'écran, qu'une fraction de seconde. Or, Marker, montre, tout aussi rapidement, pas moins de quatre autres visages, quatre autres regards à la caméra. Ce «vrai regard», fulgurant, est donc multiplié en cinq instances qui multiplient - et contredisent tout à la fois - le choc singulier, comme s'il s'agissait de représenter les variations d'une trace mnésique, cinq fois reprise (figs. 5-7). À chaque fois que ce regard rencontre celui de la caméra, le médium ne disparaît pas dans ce qu'il représente, mais se manifeste pour lui-même: en même temps que ce qu'il montre, il se montre lui-même, s'expose

17. Gilles Deleuze, Capitalisme et schizophrénie: Mille plateaux, Paris, Éditions de Minuit, coll. «Critique», 1980, p. 233.

18. Chris Marker, «Sans soleil (commentaire)», Trafic, n 6, printemps 1993, p. 85. 

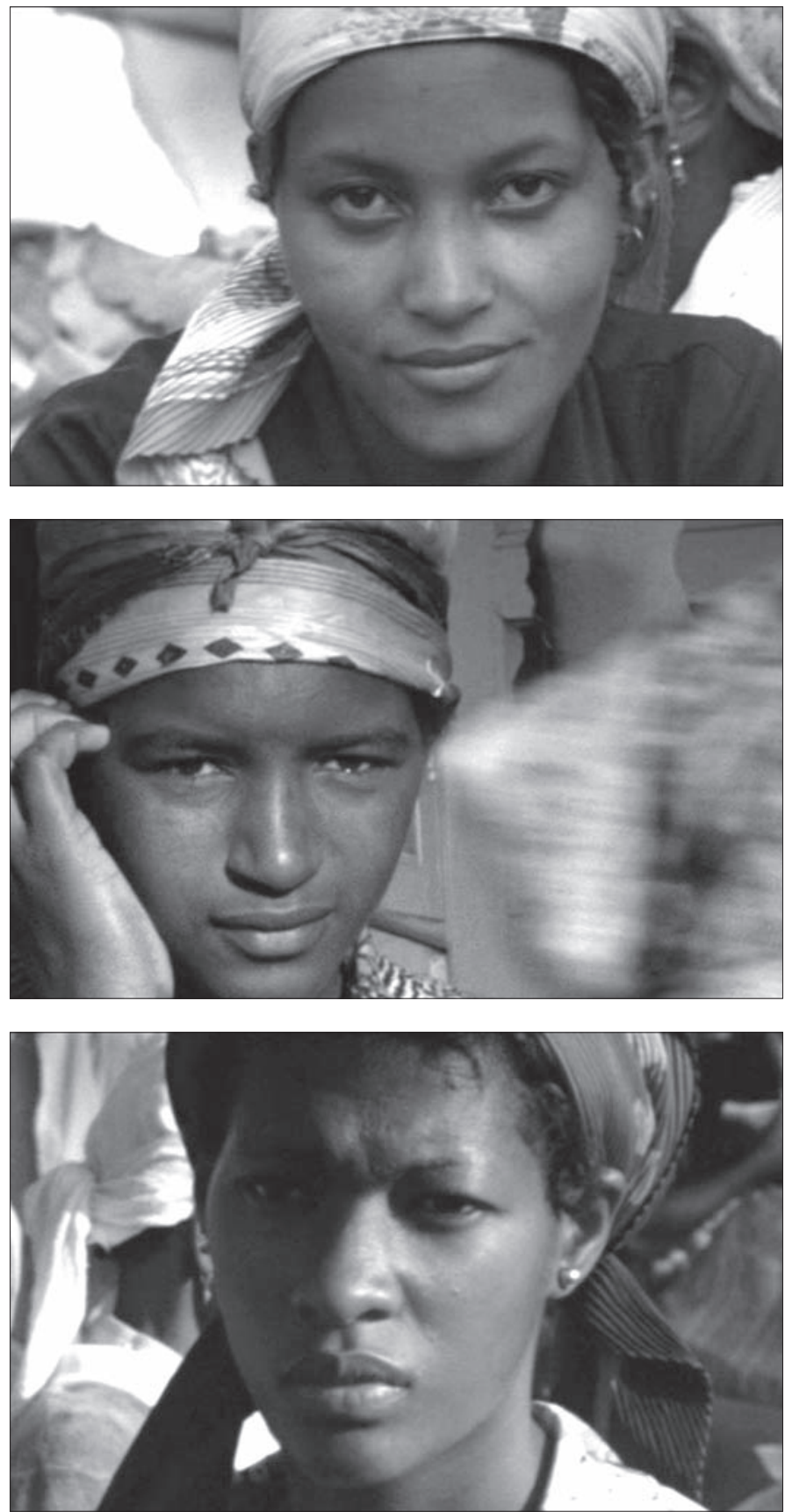

Figs. 5-7. Chris Marker, Sans soleil, 1983 (c) Argos Films. Avec l'aimable autorisation de Argos Films. 
en tant que dispositif. C'est dans cette «exposition », cette mise à l'examen du regard-caméra lui-même que la rencontre avec la (ou les) femmes de Bissau se produit, justement parce qu'il permet d'atteindre une «égalité du regard », singulière et en même temps insaisissable dans sa singularité-même.

La dernière image de Sans soleil reprend d'ailleurs un de ces visages, comme s'il était un signe-emblème pour tout le film. Cette image a été toutefois travaillée par ce synthétiseur qui, nous dit le commentaire, produit des «images moins menteuses", car «au moins elles se donnent pour ce qu'elles sont, des images, pas la forme transportable et compacte d'une réalité déjà inaccessible ${ }^{19}$ ». Cette image manipulée par la machine, immobilisée dans un moulage d'émulsion, est devenue accessible: «Il m'écrit que maintenant il peut fixer le regard de la dame du marché de Praia, qui ne durait que le temps d'une image ${ }^{2 \circ} »$. La technique permet de faire perdurer l'instant du regard, en maintenant suspendue, en même temps qu'elle le falsifie, son caractère propre: la fugacité. Maintenir l'instant du regard en tant qu'instant, comme une persistance de la perception, comme une impression de mémoire, c'est ce que nous montrait La jetée, c'est ce que réalisent à plusieurs reprises Sans soleil, Level Five, Le tombeau d'Alexandre.

L'arrêt sur image est, plus souvent qu'autrement, un arrêt sur regard, qui expose à la fois la nudité essentielle du visage et sa dissimulation, en même temps qu'il amène le dispositif filmique à se révéler. Ici comme ailleurs, le travail de Marker consiste à arrêter ces images, les ralentir, les répéter, pour les délier, montrer ce qu'elles cachent, ce qu'elles révèlent, tout en les affichant en tant qu'images.

Selon Agamben, une image travaillée par les puissances de l'arrêt et de la répétition «est un "moyen pur", qui se montre en tant que tel²1». De la même façon, les regards à la caméra forcent, d'une certaine manière, le jeu de la dissimulation à se montrer, et, « dans la mesure même où ils dénoncent la falsification, ils apparaissent plus vrais[...] ». Agamben, dans son texte «Le visage », ajoute: «ce qui reste caché n'est pas pour [l'homme] quelque chose derrière l'apparence, mais le fait même d'apparaître, le fait de n'être rien d'autre que visage ${ }^{22}$.» C'est,

19. Chris Marker, «Sans soleil», p. 86.

20. Chris Marker, «Sans soleil», p. 97.

21. Giorgio Agamben, «Le cinéma de Guy Debord» [1995], dans Image et mémoire, Paris, Desclée de Brouwer, coll. «Arts et esthétique», 2004, p. 75.

22. Giorgio Agamben, «Le visage» (1995), dans Moyens sans fins, notes sur la politique, Paris, Payot \& Rivages, coll. «Rivages poche / Petite bibliothèque », 2002, p. 107. Il est admis que ce n'est pas le même type de visage qui s'adresse à nous, dans un documentaire et dans un film de fiction. Il demeure que, fondamentalement, dans les deux cas, la caméra est reconnue comme présence, et ne disparaît pas en tant que médium. $\mathrm{La}$ «falsification» dont il est question ici n'est alors pas celle de la fiction proprement dite, mais un régime particulier de falsification markérien, qui est celui de Sans soleil, du 
en somme, cette apparence de l'apparence, qui dit la vérité du visage et, dans ce cas-ci, de sa médiation filmique, sa possibilité et sa fragilité.

\section{« LA MORT AU TRAVAIL "}

C'est à un autre niveau discursif que le dispositif est mis en scène par le visage et le regard dans Level Five. Il s'agit de la séquence des femmes de Saipei, captées par une caméra cependant qu'elles s'apprêtaient à se donner la mort en sautant en bas d'un récif. C'est en revoyant ces images, en arrêtant leur flux, que l'on peut lire cet instant fatidique où l'une des femmes, croisant le regard de la caméra et se sachant filmée, sent qu'elle doit sauter, qu'elle n'a plus le choix. Le caméraman, en somme, «l'abat, comme un chasseur». Laura, la protagoniste de Level Five, associe à cette image, par surimpression, celle d'un autre film, celui de Nicole Védrès, $1900(1948)^{23}$, où une image d'archive montre un «homme-oiseau », sur le point de tenter une expérience périlleuse en sautant du haut de la Tour Eiffel. Dans les deux cas, c'est à l'instant où le regard des protagonistes rencontre celui de la caméra que Marker fige l'image, qu'un pacte contracté avec la mort est définitivement conclu.

L'arrêt sur image révèle quelque chose de l'image, en permet une lecture, parfois explicite, parfois ambiguë. Il est assez significatif que ce soit presque toujours sur un visage, souvent en gros plan, souvent de femme, souvent sur un regard à la caméra, que l'on retrouve cette pratique chez Marker (Sans soleil en compte au moins trois ou quatre exemples). À chaque fois, il semble qu'il y ait une volonté de suspendre le temps évanescent du regard en allongeant «artificiellement» sa durée. Mais n'est-ce pas alors l'embaumer, le momifier, lui imposer un masque mortuaire dans le moulage photographique?

L'arrêt sur image fige le temps, confère à ces visages une valeur d'éternité, tout en arrachant au temps la vie du sujet qu'elle capte. Ce serait quelque chose comme une radicalisation de cette «momie du changement » dont parlait Bazin $^{24}$, et dont on trouverait plusieurs exemples dans les films de Marker. On le

sujet-filmeur-supposé, Sandor Krasna, des lettres envoyées, lues par Florence Delay, etc. On pourrait aussi se demander dans quelle mesure il s'agit d'un jeu de séduction, et qui est ce «moi» dont parle le commentaire.

23. Paris 1900 est un film composé d'images d'archives de la Belle Époque, des origines du cinéma jusqu’à la Grande guerre, hyperboliquement salué à l'époque par André Bazin, «Paris 1900. À la recherche du temps perdu », L'écran français, 30 septembre 1947, repris dans Le cinéma français de la Libération à la Nouvelle Vague (1945-1958), Paris, Éditions Cahiers du cinéma, 1998, coll. «Petite bibliothèque des Cahiers du cinéma », p. 241-243.

24. André Bazin, «Ontologie de l’image photographique» [1945], dans Qu'est-ce que le cinéma?, Paris, Cerf, 1997, p. 14. 
sait, le visage entretient un rapport précis avec le temps, dans la mesure où c'est sur lui que se lit son ouvre, entre les creux et les plis qu'il lui impose. Le temps a beau être préservé sur la pellicule, ces visages captés par la caméra exposent l'œuvre du temps, même s'ils le suspendent. Sur ces visages, surface sur laquelle se lit le passage du temps, le cinéma est noué intimement avec la mort. Et le cinéma est allé le plus loin dans son interrogation de la mort, lorsqu'il a compris que c'était là que la mort se déroulait²5.

Comme l'avance Jacques Aumont,

[...] la possibilité du visage est la possibilité de connaître sa propre mort. Le visage est l'apparence d'un sujet qui se sait humain, mais tous les hommes sont mortels: le visage est donc l'apparence d'un sujet qui se sait mortel. Ce qu'on cherche dans le visage, c'est le temps en tant qu'il signifie la $\operatorname{mort}^{26}$.

C'est en rendant le temps sensible au visage, ou le visage sensible au temps, que Marker, comme plusieurs grands cinéastes, a su filmer la mort en captant la vie. Nous pourrions dire des visages, comme de ces masques, dans Les statues meurent aussi, qu’ils «luttent avec la mort. Ils dévoilent ce qu'elle veut cacher $^{27}$. »

C'est à la faveur de cet arrêt sur image que la mort devient aussi le lieu d'une rencontre avec le visage. Comme l'exprime Aumont à la suite de Balàzs, «il faut pouvoir maintenir, ou soutenir, la contemplation, suspendre le temps (de l'action), pour mieux épouser le temps du visage, sans distance ${ }^{28} »$.

\section{VISAGE ET INTERFACE: LE TEMPS DES FANTÔMES}

Selon Deleuze, le gros plan, parce qu'il permet de rompre avec la triple fonction du visage (socialisante, individuante, communicationnelle) et le désengage de toutes ses relations, confère aux affects du visage un caractère éminemment fantomatique. «Le gros plan fait du visage un fantôme, et le livre aux fantômes ${ }^{29}$. »

25. Il suffit sans doute de penser à Nick's Movie (1980) de Wim Wenders, Dernières paroles, ma sœur Yoka [1935-1997] (1997) et Vacances prolongées (2000) de Johann van der Keuken, Near Death (1989), de Frederick Wiseman, et, pour la fiction, Cris et chuchotements (Bergman, 1975), La passion de Jeanne d'Arc (Dreyer, 1929), etc. On se rappellera aussi la phrase de Bruno Forestier (Michel Subor), dans Le petit soldat (1960) de Godard, durant la séance de photographie avec Véronika (Anna Karina) : «Elle m’a regardé d’un air angoissé et j'ai eu l'extraordinaire sensation de photographier la mort». À cet instant, au moment où Véronika croisait le regard de la caméra, Godard procédait à un arrêt sur image.

26. Jacques Aumont, Du visage au cinéma, p. 197.

27. Chris Marker, «Les statues meurent aussi» dans Commentaires, p. 18.

28. Jacques Aumont, Du visage au cinéma, p. 85.

29. Gilles Deleuze, L'image-mouvement, p. 141. 
D'ailleurs, tout medium de reproduction-communication, qu'il soit sonore ou visuel, susciterait, d'une façon ou d'une autre, selon l'auteur, des fantômes ${ }^{30}$.

Level Five - film peuplé à tous les niveaux de fantômes - exprime bien cet état du visage en gros plan, qui nous regarde et nous parle d'outre-temps, dans cette indécidabilité fantomatique de la présence et de son effacement. Le fantôme devient ici un médium, qui nous permet, dans le présent de sa manifestation, de nous connecter avec le passé. C'est ainsi que Laura ( $a$ face in the misty light»), dans Level Five, parle à la caméra en s'adressant à un autre fantôme (son compagnon disparu), qui la spectralise d'autant plus. À travers elle, c'est toute une mémoire de l'histoire passée (collective et singulière), qui hante encore le présent, que l'on traverse, par témoignages interposés et images superposées. Ce serait peut-être un troisième mode du visage, après le mode narratif et le mode spectaculaire: le mode spéculaire, qui s'impose en flottant dans cet entre-temps, cet entre-deux du monde. Dans Level Five, ce mode spéculaire du visage apparaît en tant qu'inter-face.

Comme dans un journal filmé, Laura, personnage principal de Level Five, s'adresse directement à une caméra vidéo. C'est un des traits frappants de ce film, de tourner tout entier autour d'un visage qui assure la liaison avec les autres niveaux du film et nous connecte avec d'autres visages : les témoins, les généraux japonais et américains, les passants de la ville de Tokyo, les visages numérisés, la «galerie des masques », etc. Les couches successives de fiction et de traces documentaires, de niveaux discursifs et de supports médiatiques qui s'emboîtent dans ce film en font un véritable palimpseste filmique auquel les visages participent activement.

Ce film offre, en quelque sorte, un face à face avec l'inter-face, lieu d'inscription et de médiation (informatique et symbolique) qui se maintient en tant que moyen. Le visage de Laura, comme l'interface de l'ordinateur - le jeu de la bataille d'Okinawa, le réseau O.W.L. - est à la fois un organe récepteur et un écran-surface de projection (comme ce visage en pierre blanc qui apparaît à plusieurs moments du film). C'est en passant par cette interface que le film assure ses différents raccords, se met et nous met en réseau ${ }^{31}$. Le visage est alors un moyen, un milieu de médiation de l'expression et de l'expérience, qui fait converger une

30. C'est la distinction de Kafka, que Deleuze reprend à son compte, entre les moyens de communication-translation et de communication-expression (lettre, téléphone, radio, etc.) qui «suscitent les fantômes sur notre route et nous dévient vers des affects incontrôlés». (Gilles Deleuze, L'image-mouvement, p. 142)

31. "The concept of the interface comes to define, both figuratively and literally, the machinic connectivity of digital culture. » (David N. Rodowick, Reading the Figural, or, Philosophy After the New Media, Durham \& London, Duke Univ. Press, 2001, p. 214) 
multiplicité de séries divergentes et de supports hétéronomes (film, images numériques, peinture, images vidéo, images télévisuelles, dessins animés, etc.).

Les nouvelles technologies de l'information et des communications ont provoqué des mutations profondes dans notre façon de concevoir l'identité ainsi que les politiques et l'économie identitaires. Level Five informe en quelque sorte ce nouvel état de fait, où la surveillance à outrance de l'individu se confond avec l'anonymat toujours plus grand des populations. Il témoigne de la profusion des images qui tapissent notre environnement, tout en fournissant des axes de lecture critique qui appellent un arrêt du flux des images, qui nous permettent de revoir ce que nous croyions avoir vu, ce que nous croyions connaître, parmi elles, les images de guerre. Marker en expose la nature, en en refaisant l'histoire. Il s'interroge sur les nouveaux moyens de médiation avec le passé qu'ont rendu possibles les nouvelles technologies, tout en montrant les leurres et les dérives de la médiatisation ${ }^{2}$.

Les visages sont de plain-pied dans cette recherche. Ils se montrent souvent en images de synthèse, pixellisés, formés sur l'écran de l'ordinateur, présentés dans leur existence technologique. Sur le réseau, Laura se promène dans la galerie des masques où, comme ailleurs, le visage ne se révèle que dans la mesure où il s'affiche dissimulé, où il expose sa pâte feuilletée, ses pelures d'oignons (dirait Rilke). C'est ce que prétendait Agamben:

le visage découvre seulement dans la mesure où il dissimule et dissimule dans la mesure même où il découvre [...] Parce que le visage n’est que le lieu de la vérité, il est immédiatement le lieu d'une simulation et d'une impropriété irréductible 33.

Le visage de Laura, dont le nom auratique est emprunté à une histoire de fantômes (Laura, Otto Preminger, 1944), est lui-même un palimpseste qui dissimule en même temps qu'il révèle, qui semble se modifier à chaque apparition (chevelure différente, teint modifié, autre éclat dans les yeux). Laura joue dans ce film, comme Chris Marker, un jeu de dissimulation et de révélation. Elle s'exprime en s'abîmant, en perdant ses coordonnées individuelles, sa spécificité («mon drame le plus intime, est aussi le plus banal»). De la sorte, il n’est pas possible de dire que le visage de Laura dans Level Five traduit un processus d'individuation; plutôt, il ne cesse d'être traversé par autre chose : il est montré avec d'autres images, d'autres visages, superposé à des lignes de codes informatiques, reflété dans l'écran, recouvert par des masques, etc. Il a beau être toujours là, il ne cesse de fuir, ses traits ne cessent de se défaire. D’ailleurs, au bout du film,

32. Sur cette distinction, voir Johanne Villeneuve et Brian Neville, «In Lieu of Waste», dans Waste-Site Stories, p. 1-29.

33. Giorgio Agamben, «Le visage», p. 106. 
son visage disparaîtra, littéralement floué ( mis au flou) par le zoom, par un excès du gros plan. Son visage perd ses traits, ne devient plus qu'une surface blanche et indistincte, une trace de l'oubli : «le gros plan visage est à la fois la face et son effacement ${ }^{34}$. »

\section{RENDRE LE VISAGE}

C'est sans doute par là qu'il nous faut passer pour atteindre une vérité du visage, qui ne semble pouvoir devenir le principe d'unité du film qu'en acceptant de perdre ses traits, d'être dévisagé ou traversé par autre chose que lui. Rendre le visage, c'est en montrer la multiplicité, la fragilité, l'inquiétude, la perte. Pour parler comme Agamben, on dira que « saisir la vérité du visage signifie appréhender non pas la ressemblance, mais la simultanéité des faces, la puissance inquiète qui les maintient ensemble et les unit35».

La figure du visage chez Marker est apparue sous une multiplicité de modes: comme image-souvenir, comme signe de mémoire et signe d'une mémoire, comme nœud gordien de certains enjeux de figurations du dispositif, et de la relation de ce dispositif au temps et à la mort; enfin, comme interface, surface d'interrogation et de projection des nouvelles technologies, ainsi que des modalités repensées de la relation entre histoire et mémoire. Le visage a pu apparaître comme une figure de médiation privilégiée dans son œuvre, puisqu'en lui se délient ses questions les plus fondamentales: la mémoire d'un objet perdu, une réflexion sur la non-transparence du médium et de la vérité à contre-temps des images, une éthique du deuil sous le signe, toujours reconduit, de la non-réconciliation.

34. Gilles Deleuze, L'image-mouvement, p. 142.

35. Giorgio Agamben, «Le visage», p. 112. 